\title{
Current-induced magnetic vortex core switching in a Permalloy nanodisk
}

Y. Liu, S. Gliga, R. Hertel, and C. M. Schneider

Citation: Appl. Phys. Lett. 91, 112501 (2007); doi: 10.1063/1.2780107

View online: https://doi.org/10.1063/1.2780107

View Table of Contents: http://aip.scitation.org/toc/apl/91/11

Published by the American Institute of Physics

\section{Articles you may be interested in}

Dynamics of vortex core switching in ferromagnetic nanodisks

Applied Physics Letters 89, 262507 (2006); 10.1063/1.2424673

Eigenfrequencies of vortex state excitations in magnetic submicron-size disks

Journal of Applied Physics 91, 8037 (2002); 10.1063/1.1450816

A frequency-controlled magnetic vortex memory

Applied Physics Letters 96, 132506 (2010); 10.1063/1.3373833

The design and verification of MuMax3

AIP Advances 4, 107133 (2014); 10.1063/1.4899186

Electric-current-driven vortex-core reversal in soft magnetic nanodots

Applied Physics Letters 91, 082506 (2007); 10.1063/1.2773748

Switching magnetic vortex core by a single nanosecond current pulse

Applied Physics Letters 93, 152502 (2008); 10.1063/1.3001588

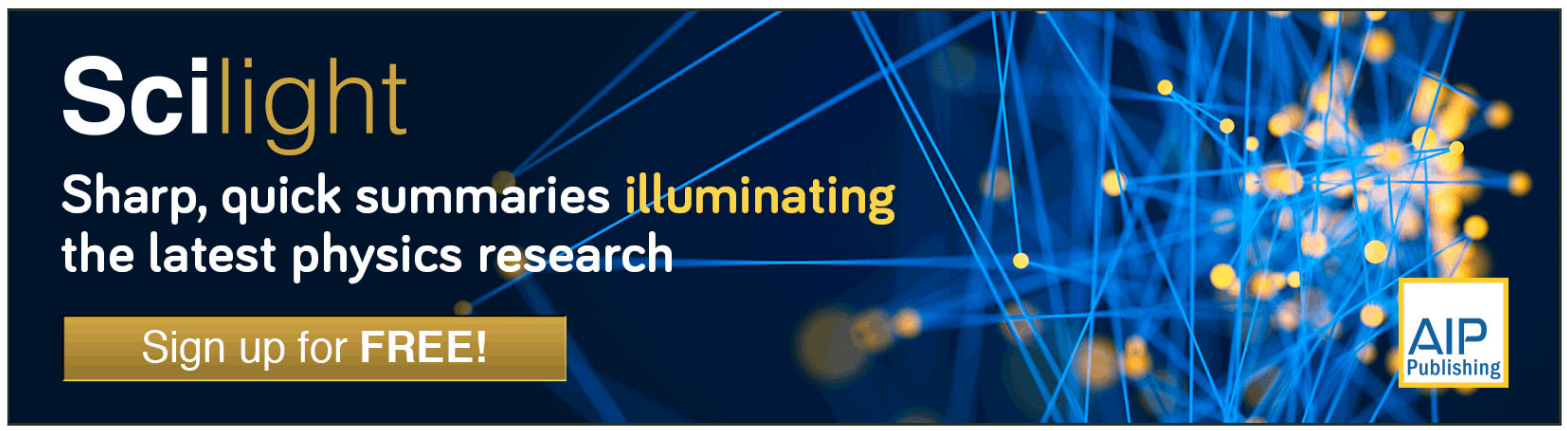




\title{
Current-induced magnetic vortex core switching in a Permalloy nanodisk
}

\author{
Y. Liu \\ Institut für Festkörperforschung IFF-9 "Elektronische Eigenschaften,” Forschungszentrum Jülich GmbH, \\ D-52425 Jülich, Germany and Department of Physics, Tongji University, 200092 Shanghai, People's \\ Republic of China \\ S. Gliga, R. Hertel, ${ }^{\text {a) }}$ and C. M. Schneider \\ Institut für Festkörperforschung IFF-9 "Elektronische Eigenschaften," Forschungszentrum Jülich GmbH, \\ D-52425 Jülich, Germany
}

(Received 2 February 2007; accepted 25 July 2007; published online 10 September 2007)

\begin{abstract}
The authors report on the switching of a magnetic vortex core in a submicron Permalloy disk, induced by a short current pulse applied in the film plane. Micromagnetic simulations including the adiabatic and nonadiabatic spin-torque terms are used to investigate the current-driven magnetization dynamics. They predict that a core reversal can be triggered by current bursts a tenth of a nanosecond long. The vortex core reversal process is found to be the same as when an external field pulse is applied. The control of a vortex core's orientation using current pulses introduces the technologically relevant possibility to address individual nanomagnets within dense arrays. (C) 2007 American Institute of Physics. [DOI: 10.1063/1.2780107]
\end{abstract}

Patterned soft-magnetic thin film elements with lateral extension above a critical size naturally form flux-closure patterns, ${ }^{1}$ which contain magnetic vortices, i.e., small regions, where the magnetization direction circulates in the film plane around a nanometer-sized core. ${ }^{2}$ At the center of this core, the magnetization points perpendicular to the plane. ${ }^{3}$ The study of the static and dynamic properties of vortices and vortex cores in particular has recently emerged as a dynamic field of investigation. ${ }^{2-8}$ Magnetic vortex cores have indeed been considered as possible candidates for magnetic data storage ${ }^{9}$ due to several attractive properties: their size is of only a few tens of nanometers, ${ }^{2}$ they exhibit perfect bistability (pointing either upwards or downwards, defining the vortex polarization), and they form naturally and ultimately have a high thermal stability. All these features represent important prerequisites for possible applications to data storage. In order to store information using a vortex core, mechanisms for a controlled switching of its orientation are required. The direct reversal of vortex cores by means of an applied field oriented perpendicular to the plane requires large field values of more than $200 \mathrm{mT}^{9}{ }^{9}$ which gives an idea of the high stability of these structures. Recently, it has been demonstrated that the vortex core could be switched by means of sinusoidal low field pulses applied in the plane of the sample. ${ }^{6}$ In this case, the gyrotropic resonance was exploited-a low-frequency mode where the vortex rotates around its equilibrium position. ${ }^{5,7,8,10}$ In order to use the resonant properties of the vortex, it has to be brought on its gyrotropic orbit. The time required for this depends on the gyrotropic frequency, which is approximately proportional to the sample's aspect ratio and has typical values of about a gigahertz or below for thin-film elements. ${ }^{7}$ In practice, the switching time can be defined as the time between the application of the field and the completion of the core switch. In this sense, the switching time of the resonant scheme is of the order of nanoseconds. Based on micromagnetic simulations, a faster route to achieve core reversal has been pro-

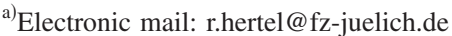

posed recently. ${ }^{11,12}$ In this case, which does not use the gyrotropic resonance, the reversal is induced by a suitably shaped unipolar in-plane magnetic field pulse only a few picoseconds long. The required amplitude of the field pulse is larger [approximately $70 \mathrm{mT}$ (Ref. 11)] than in the case of resonant switching (approximately $2 \mathrm{mT},{ }^{6}$ ) but this switching scheme is much faster. In spite of their respective advantages (low fields, high speed) both the resonant and the nonresonant switching modes exhibit a common problem in terms of applicability: the lack of selectivity of the individual elements. Reliably addressing a single nanodisk inside a dense array is very difficult using external fields. In this letter, we present a fast and simple method to switch magnetic vortex cores by applying short electric current pulses, only $100 \mathrm{ps}$ long. The electric current pulse is applied in the plane of the element. We thus show that a fast toggle core switching mechanism can be triggered in a relatively simple way which is compatible with integrated circuits, thereby solving the issue of selectivity.

The results were obtained using micromagnetic finiteelement simulations based on the Landau-Lifshitz-Gilbert equation. Our micromagnetic code used in previous simulations ${ }^{13}$ has been extended to consider the spin torque exerted by an electric current flowing through the sample. This effect of current-driven magnetization dynamics is modeled by including the adiabatic and the nonadiabatic spin torque terms $^{14,15}$ into the Gilbert equation,

$$
\begin{aligned}
\frac{d \boldsymbol{m}}{d t}= & -\gamma \boldsymbol{m} \times \boldsymbol{H}_{\mathrm{eff}}+\alpha \boldsymbol{m} \times \frac{d \boldsymbol{m}}{d t}-(\boldsymbol{u} \nabla) \boldsymbol{m} \\
& +\beta \boldsymbol{m} \times[(\boldsymbol{u} \nabla) \boldsymbol{m}],
\end{aligned}
$$

where $\boldsymbol{m}=\boldsymbol{M} / M_{s}$ is the normalized local magnetization $\left(M_{s}\right.$ the saturation magnetization), $\boldsymbol{H}_{\text {eff }}$ is the effective field containing the exchange and the dipolar field, $\beta$ is a dimensionless parameter describing the strength of the nonadiabatic term, and $\alpha$ is the Gilbert damping constant. The vector $\boldsymbol{u}$ is pointing parallel to the electron flow direction and has an amplitude of $u=j P g \mu_{B} /\left(2 e M_{s}\right),{ }^{15}$ where $j$ is the current density, $P$ is the degree of electron polarization, $g$ is the Landé 


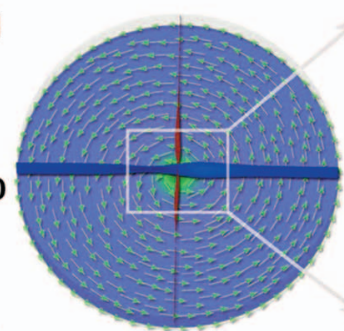

Static equilibrium

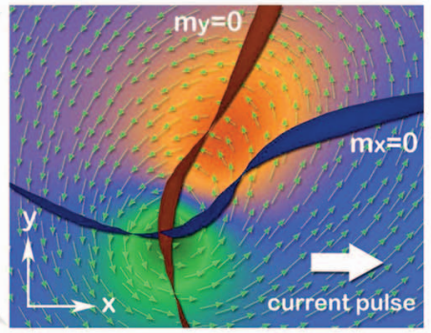

430 ps

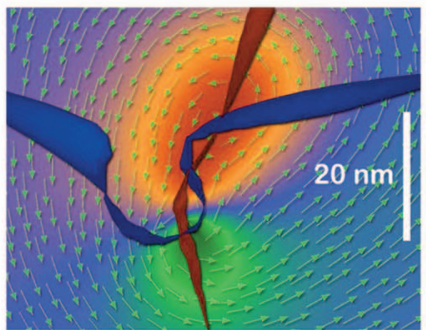

466 ps

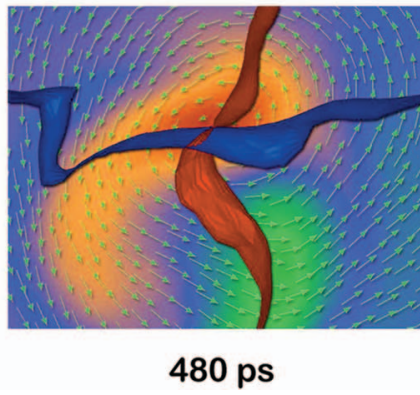

FIG. 1. (Color) Current-induced vortex core reversal in a Py nanodisk of $200 \mathrm{~nm}$ diameter and $20 \mathrm{~nm}$ thickness. A Gaussian current pulse is applied in the sample plane with a strength of $7.4 \times 10^{12} \mathrm{~A} / \mathrm{m}^{2}$ and a width $\sigma=100 \mathrm{ps}$. The magnetic vortex structure at equilibrium is shown on the left. In the three frames on the right, the evolution of the magnetization is shown for a small region around the vortex core, where a vortex-antivortex pair nucleates. As the vortex is shifted in the direction of the electron flow prior to the pair creation, these frames are not taken in the same areas of the sample. The arrows represent the in-plane magnetization direction, while the color code displays the $z$ component of the magnetization. The blue and red ribbons are the $m_{x}=0$ and $m_{y}=0$ isosurfaces, respectively. The time is measured from the moment the pulse is applied (see Fig. 2).

splitting factor, $\mu_{B}$ is the Bohr magneton, and $e$ is the electron charge. As a model system, we consider a diskshaped Permalloy (Py) sample of $d=200 \mathrm{~nm}$ diameter and $t=20 \mathrm{~nm}$ thickness, as used in Ref. 11. The parameters used in the simulation are $A=13 \mathrm{pJ} / \mathrm{m}$ (exchange constant), $\mu_{0} M_{s}=1.0 \mathrm{~T}, \alpha=0.03, P=0.7, \beta=0.02,{ }^{15} K=0$ (anisotropy constant). The sample is discretized into approximately 216000 irregular tetrahedral elements, corresponding to a cell size of about $3 \mathrm{~nm}$. A homogeneous current density distribution is assumed, as was done recently in a similar study by Kasai et al., ${ }^{16}$ where the resonant, current-pulse induced gyrotropic vortex core motion was investigated.

The current-induced vortex core reversal is studied for short Gaussian-shaped current pulses $(\sigma=100 \mathrm{ps})$ of varying strengths. In all cases, the maximum strength is reached at 300 ps. To obtain a core reversal, we found that for the considered sample the amplitude of these pulses must exceed a minimum value of $j=6.7 \times 10^{12} \mathrm{~A} / \mathrm{m}^{2}$. Although such a high current density might endanger the structural stability of the sample if it was applied continuously, the damaging effects of the current should be small in the present case where only ultrashort pulses are used.

A typical example of the vortex core reversal process is shown in Fig. 1, starting with a vortex whose core points in the positive $z$ direction. The micromagnetic processes leading to the vortex core reversal are identical to the ones described in Ref. 11, where field pulses were used. An isosurface representation as introduced in Ref. 17 is used to precisely locate the vortex core. The in-plane magnetization is first heavily distorted and a pronounced "dip" is formed $^{16,18}$ as a result of the current running through the sample. About 460 ps after the application of the pulse (i.e., 160 ps after the maximum has been reached), the distortion leads to the creation of a vortex-antivortex pair, which can unambiguously be recognized by the two additional crossings of the $m_{x}=0$ and $m_{y}=0$ isosurfaces. Both cores of the new pair are pointing in the opposite $z$ direction of the initial core. 11 The newly formed antivortex and the oppositely polarized initial vortex subsequently annihilate. ${ }^{f}$ The latter subprocess unfolds over approximately $10 \mathrm{ps}$ and leaves a single vortex core, which is oppositely polarized with respect to the initial one. The formation of vortex-antivortex pairs after application of short current pulses is consistent with recent experimental observations by Kläui et al. ${ }^{19}$

Figure 2 shows the evolution in time of the sample's spatially averaged energy density in the case of an applied current pulse of $7.4 \times 10^{12} \mathrm{~A} / \mathrm{m}^{2}$ and $\sigma=100 \mathrm{ps}$. The exchange energy rapidly decreases after the annihilation of the vortex-antivortex pair, leading to a sudden drop in the total energy. ${ }^{11}$

By increasing the pulse strength, it is possible to produce multiple switches, as shown in Fig. 3. Multiple switches are a repeated series of vortex-antivortex pair creation and annihilation processes. In all cases, the total energy is seen to immediately decrease following each core reversal. However, if the energy supplied by the current pulse after a core reversal is sufficient to overcompensate dissipation, the energy can still increase before further switches occur. A double core switching is obtained with current pulses of about $8.5 \times 10^{12} \mathrm{~A} / \mathrm{m}^{2}$, while triple and quadruple switches occur with pulses of $10 \times 10^{12}$ and $11 \times 10^{12} \mathrm{~A} / \mathrm{m}^{2}$, respectively. Ultimately, for very large currents (above 20 $\times 10^{12} \mathrm{~A} / \mathrm{m}^{2}$ ), the vortex core is expelled from the sample. ${ }^{20}$

The diagram in Fig. 3(b) shows the number of core switches as a function of the applied current's strength. Clear "steps" are observed. While the core reversal mechanism is mediated by the formation of a vortex-antivortex pair, it is the annihilation process which leaves the vortex with an oppositely polarized core. As shown in Ref. 17, such an annihilation is mediated by a magnetic singularity (Bloch point) which is injected through the sample. Since the energy of formation of a Bloch point is uniquely a function of the

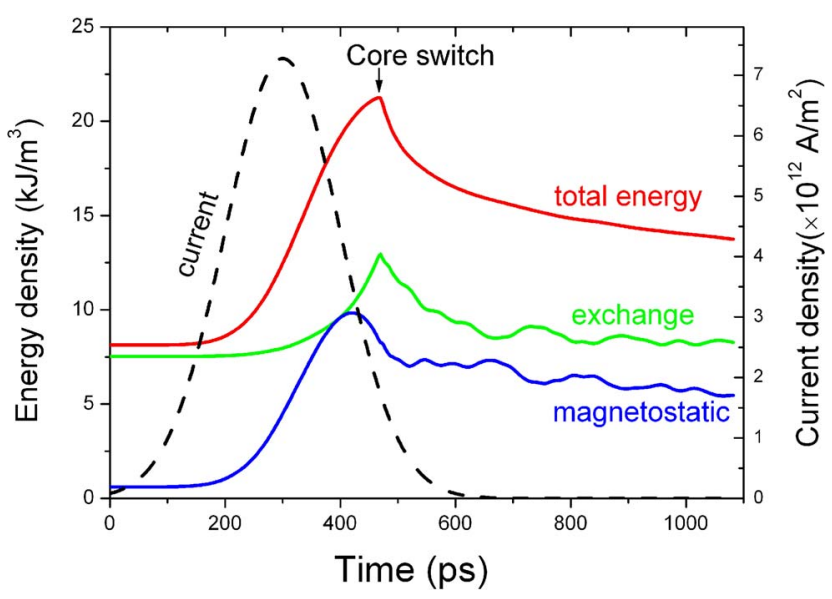

FIG. 2. Evolution as a function of time of the total, magnetostatic, and exchange energy densities in the Py disk following a current pulse of a duration of $\sigma=100 \mathrm{ps}$ and a peak value of $7.4 \times 10^{12} \mathrm{~A} / \mathrm{m}^{2}$. 

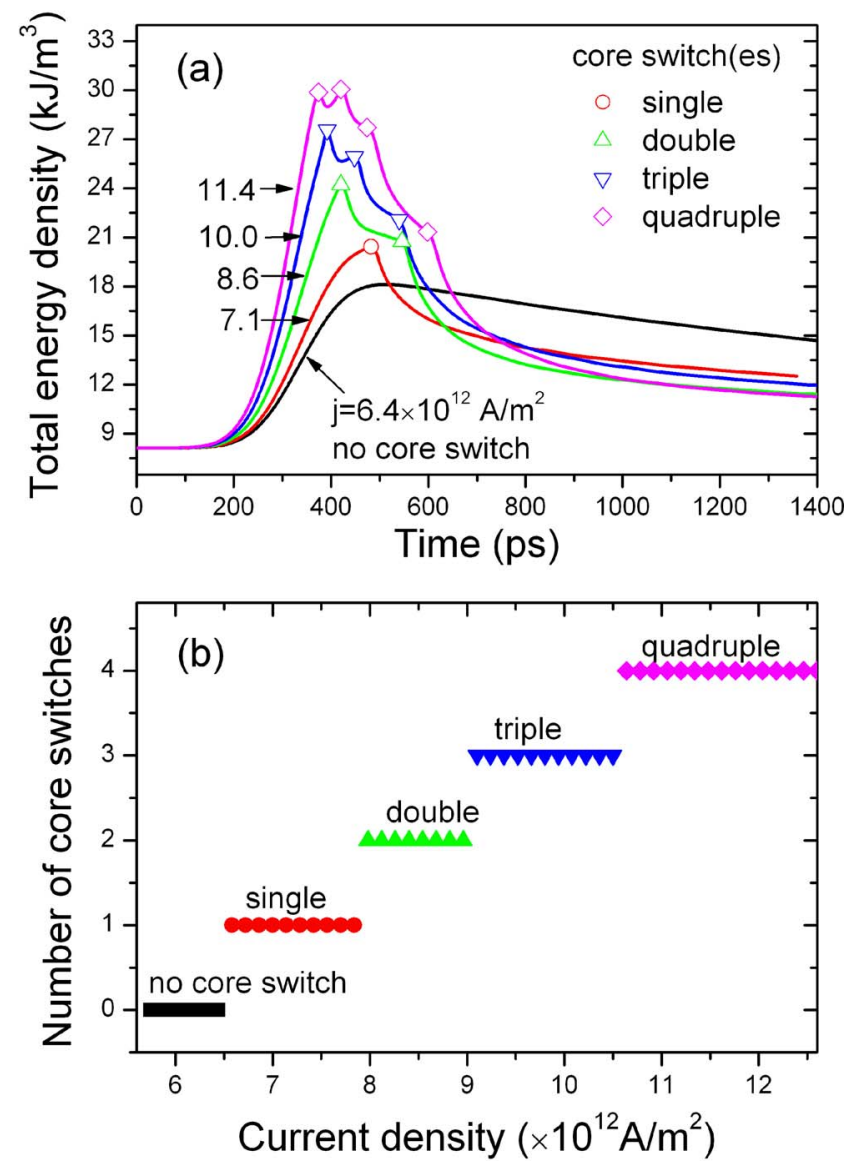

FIG. 3. (a) Total energy as a function of time for multiple core switches. (b) Number of times the vortex core switches depending on the applied current density.

exchange constant, ${ }^{21}$ the annihilation (and thus the reversal) process can only occur at specific energy values, resulting in the observed steps.

In conclusion, we have presented the possibility of reversing the polarization of a magnetic vortex core using short current pulses. The magnetization reversal process, which consists of a complicated sequence of vortex-antivortex pair creation and annihilation events, unfolds on a time scale of approximately $40 \mathrm{ps}$, i.e., shorter than the duration of the pulses applied in this study. Further investigations are required to explore the limits of the operational range for a controlled, single toggle switching in terms of pulse duration and pulse strength, and to determine how short a current pulse can be to trigger a vortex core reversal. The currentinduced vortex core reversal opens the possibility of addressing individual magnetic elements in a vortex state within an array of nanoelements. This feature could make vortex cores interesting candidates for data storage purposes in future devices.

After submission of this letter, experimental evidence for current-driven vortex core switching has been reported by Yamada et $a l^{22}$

One of the authors (Y.L.) acknowledges financial support from the Alexander von Humboldt Foundation.

${ }^{1}$ R. P. Cowburn, D. K. Koltsov, A. O. Adeyeye, M. E. Welland, and D. M. Tricker, Phys. Rev. Lett. 83, 1042 (1999).

${ }^{2}$ A. Wachowiak, J. Wiebe, M. Bode, O. Pietzsch, M. Morgenstern, and R. Wiesendanger, Science 298, 577 (2002).

${ }^{3}$ T. Shinjo, T. Okuno, R. Hassdorf, K. Shigeto, and T. Ono, Science 289 , $930(2000)$

${ }^{4}$ S. B. Choe, Y. Acremann, A. Scholl, A. Bauer, A. Doran, J. Stohr, and H. A. Padmore, Science 304, 420 (2004).

5 J. P. Park, P. Eames, D. M. Engebretson, J. Berezovsky, and P. A. Crowell, Phys. Rev. B 67, 020403(R) (2003).

${ }^{6}$ B. Van Waeyenberge, A. Puzic, H. Stoll, K. W. Chou, T. Tyliszczak, R. Hertel, M. Fähnle, H. Brückl, K. Rott, G. Reiss, I. Neudecker, D. Weiss, C. H. Back, and G. Schütz, Nature (London) 444, 461 (2006).

${ }^{7}$ K. Y. Guslienko, B. A. Ivanov, V. Novosad, Y. Otani, H. Shima, and K. Fukamichi, J. Appl. Phys. 91, 8037 (2002).

${ }^{8}$ V. Novosad, M. Grimsditch, K. Y. Guslienko, P. Vavassori, Y. Otani, and S. D. Bader, Phys. Rev. B 66, 052407 (2002).

${ }^{9}$ N. Kikuchi, S. Okamoto, O. Kitakami, Y. Shimada, S. G. Kim, Y. Otani, and K. Fukamichi, J. Appl. Phys. 90, 6548 (2001).

${ }^{10}$ B. E. Argyle, E. Terrenzio, and J. C. Slonczewski, Phys. Rev. Lett. 53, 190 (1984).

${ }^{11}$ R. Hertel, S. Gliga, M. Fähnle, and C. M. Schneider, Phys. Rev. Lett. 98, 117201 (2007)

${ }^{12}$ Q. F. Xiao, J. Rudge, B. C. Choi, Y. K. Hong, and G. Donohoe, Appl. Phys. Lett. 89, 262507 (2006).

${ }^{13}$ R. Hertel, W. Wulfhekel, and J. Kirschner, Phys. Rev. Lett. 93, 257202 (2004).

${ }^{14}$ S. Zhang and Z. Li, Phys. Rev. Lett. 93, 127204 (2004).

${ }^{15}$ A. Thiaville, Y. Nakatani, J. Miltat, and Y. Suzuki, Europhys. Lett. 69, 990 (2005)

${ }^{16}$ S. Kasai, Y. Nakatani, K. Kobayashi, H. Kohno, and T. Ono, Phys. Rev. Lett. 97, 107204 (2006).

${ }^{17}$ R. Hertel and C. M. Schneider, Phys. Rev. Lett. 97, 177202 (2006).

${ }^{18}$ V. Novosad, F. Y. Fradin, P. E. Roy, K. S. Buchanan, K. Y. Guslienko, and S. Bader, Phys. Rev. B 72, 024455 (2005).

${ }^{19}$ M. Kläui, M. Laufenberg, L. Heyne, D. Backes, U. Rüdiger, C. A. F. Vaz, J. A. C. Bland, L. J. Heyderman, S. Cherifi, A. Locatelli, T. O. Mentes, and L. Aballe, Appl. Phys. Lett. 88, 232507 (2006).

${ }^{20} \mathrm{We}$ have tested that the switching mechanism is not sensitive to the choice of the material parameters $\alpha$ and $\beta$. The core reversal occurs in the same way also for lower (0.01) and higher ${ }^{11}$ values of $\alpha$. The current densities required for switching are slightly decreased as the damping is lowered, but the switching time remains unaffected. Similarly, the choice of $\beta$ is not critical. In fact, setting $\beta$ to zero yields the same result. Thus, the nonadiabatic term has negligible influence here.

${ }^{21}$ O. A. Tretiakov and O. Tchernyshyov, Phys. Rev. B 75, 012408 (2007).

${ }^{22}$ K. Yamada, S. Kasai, Y. Nakatani, K. Kobayashi, H. Kohno, A. Thiaville, and T. Ono, Nat. Mater. 6, 269 (2007). 